\title{
$\begin{array}{ll}\text { Research Square } & \text { Preprints are preliminary reports that have not undergone peer review. } \\ \text { They should not be considered conclusive, used to inform clinical practice, } \\ \text { or referenced by the media as validated information. }\end{array}$
}

\section{Transcriptome and De Novo Analysis of Rosa xanthina f. spontanea in Response to Cold Stress}

\author{
Defeng Zhuang \\ Inner Mongolia University for Nationalities \\ $\mathrm{Ce} \mathrm{Ma}$ \\ Liaoning Academy of Agricultural Sciences \\ Li Xue \\ Shenyang Agricultural University \\ Zhen Li \\ Liaoning Academy of Agricultural Sciences

\section{Cheng Wang} \\ Inner Mongolia University for Nationalities \\ Jiajun Lei \\ Shenyang Agricultural University \\ Xingfu Yuan ( $\sim$ laasy@189.cn ) \\ Liaoning Academy of Agricultural Sciences
}

Research Article

Keywords: Rose, Low-temperature stress, DEGs, Metabolic pathway

Posted Date: February 16th, 2021

DOI: https://doi.org/10.21203/rs.3.rs-199455/v1

License: (c) (1) This work is licensed under a Creative Commons Attribution 4.0 International License. Read Full License

Version of Record: A version of this preprint was published at BMC Plant Biology on October 15th, 2021. See the published version at https://doi.org/10.1186/s12870-021-03246-5. 


\section{Abstract}

Background: Rose is one of most popular ornamental plants all over the world, it is of high economical value and great cultural importance. However, chilling damage restricts its application in cold area. To elucidate the metabolic response under low temperature stress, we conducted transcriptome and De novo analysis in Rosa xanthina f. spontanea.

Results: A total of 124,106 unigenes from 9 databases were generated by De novo assembly, with mean length of $661 \mathrm{bp}$ under $4^{\circ} \mathrm{C}$ and $-20^{\circ} \mathrm{C}$ stress $\left(23^{\circ} \mathrm{C}\right.$ as control). Functional annotation and prediction of 55,084 unigenes were detected and $67.72 \%$ of these unigenes had significant similarity (Blast, $\left.\mathrm{E} \leq 10^{-5}\right)$ in the public databases. $468 \mathrm{DEGs}$ were involved in three groups: biological process (64.84\%), cellular component (9.38\%) and molecular function (25.78\%), KEGG analysis suggested that metabolic pathway, response to plant pathogen interaction, starch and sucrose metabolism, circadian rhythm plant and photosynthesis antenna proteins were significantly enriched under cold stress.

Conclusions: Our study first reported response to cold stress at the transcriptome level in Rosa xanthina f. spontanea, which can provides a theoretical basis for further studies on the molecular mechanism of cold-resistance in rose. The results was shown that the expression trend of eight DEGs selected by qRT-PCR analysis at random was consistent with the results of high-throughput sequencing.

\section{Background}

Rose is one of the most ornamental plants, which has high economical value, is very popular with people worldwide. But, due to lack of cold-tolerance, its planting application was affected by low-temperature in cold region. It was a complex metabolic process on the formation of cold-resistance in woody plants. Generally, plant growth and development were stopped when winter arrives, cold-tolerance and dormancy ability gradually formed [1]. And in this process, it will result in variation of morphological traits at transcriptional/biochemical level, and then to improve the stability of membrane system, which can be survived safely in winter, such as most temperate woody plants [2].

A transcriptome is a specific tissue or cell at a certain stage of development or the sum of all RNA that was transcribed in a functional state, mainly including mRNA and non-coding RNA [3]. Expression of the whole genome was revealed by abiotic stress tolerance at the whole transcriptional level. It is of great significance to construct the transcriptional regulatory network of abiotic stress genome in term of complex regulatory network involved in increasing abiotic stress adaptation and tolerance[4, 5]. Transcriptome sequencing has already been developed under low-temperature stress in Camellia sinensis, Populus euphratica [6, 7].

Recently, there are many studies on the transcriptome in rose. Abundant genetic information has been obtained in resistance and development with respect to transcriptome sequencing by using root, leaf, flower and fruit of rose as materials [8-11]. The fusion strategy combined with genome and proteome can be provided a certain theoretical basis for resolving the biological problems of rose in future. Transcriptome sequencing analysis had been studied under cold stress with leaves in R. multiflora [12], fruit in blueberry [13] and floral buds in $R$. hybrida [14]. So far, the transcriptome information has not been clarified because of its complexity of the formation of cold-resistance mechanisms.

R.xanthina, a wild species of Sect. Pimpinellifoliae belongs to Rosa, which is native to northeast China and west China. In addition, $R$. xanthina f. spontanea has with high cold /drought-tolerance and disease-resistance, is an important germplasm resource in breeding of modern rose [15]. In the present study, we carried out the transcriptome sequencing analysis of $R$. xanthina f. spontanea under low-temperature stress, then to clarify the function and metabolic pathway associated with DEGs, which can provide a certain theoretical foundation for cold-resistance mechanism in rose.

\section{Results}

\section{Transcriptome sequencing and assembly}


The original data obtained by sequencing with Illumina Hiseq 4000 were transformed into raw reads by base calling. The total base number obtained from nine libraries was $64.52 \mathrm{G}$ and the total number of nucleotides was between $5.23 \mathrm{G}$ and

$7.59 \mathrm{G}$ in each sample. The Q30 ratio of each sample was greater than $94 \%$ and GC content was relatively consistent, around $47 \%$ (Additional file 1). A total of 124,106 transcripts and 55,084 non-redundant unigenes were obtained from nine databases, respectively. The average length of unigenes was $661 \mathrm{bp}$ and length of N50 was $1470 \mathrm{bp}$. Consequently, the sequencing data quality was high and could meet the requirements of subsequent analysis. There were 29,582 unigenes with the length of 200-500bp accounting for $53.70 \%, 10,103$ unigenes of length $500-1000$ bp accounting for $18.34 \%, 10,112$ unigenes ranged from 1000 to $2000 \mathrm{bp}$ accounting for $18.35 \%$, and 5,287 unigenes of length more than $2000 \mathrm{bp}$ accounting for $9.6 \%$, respectively (Fig. 1).

\section{Functional annotation of unigenes}

Based on Nr, Swiss-prot (a manually annotated and reviewed protein sequence database), Pfam (protein family), KOG (Clusters of Orthologous Groups of proteins), KEGG and GO databases, which were used to annotate all unigenes with comprehensive gene function information. In the present study, a total of 37,303 unigenes were successfully annotated in $R$. xanthina f. spontanea database, which were shown all of $67.72 \%(55,084)$. Furthermore, Fragaria_vesca presented the highest frequency in the annotation results with a total of 19,964 comments, accounting for $53.52 \%$ of all the sequences, followed by Nelumbo nucifera (9.0\%), Prunus persica (3.27\%), Phaseolus vulgaris (3.13\%), Vitis vinifera (2.78\%) and Prunus mume (2.36\%)(Fig. 2 and Additional file 2).

A total of $31,258(56.75 \%)$ unigenes were assigned to $\mathrm{GO}$ terms in the cellular component, molecular function and biological process categories that were further classified into $50 \mathrm{GO}$ terms (Fig. 3 and Additional file 3).

Within the cellular component, a total of $22,417 \mathrm{DEG}$ were assigned under $4^{\circ} \mathrm{C}$ and $-20^{\circ} \mathrm{C}$ stress $\left(23^{\circ} \mathrm{C}\right.$ as the control), which indicated the union of all the DEGs were mainly related to the nucleus, cytoplasm, integral component of membrance and chloroplast. With regard to the molecular function, most of the DEGs were enriched for molecular function, protein binding and ATP binding. In the biological process, biological process, regulation of transcription and DNA-template were mainly involved.

To identify the metabolic pathways during cold stress of $R$. xanthina f. spontanea leaves, 21,992 DEGs were mapped to the KEGG database, and the top 19 KEGG pathways significantly ( $p$-value < 0.05) enriched were shown in Fig. 4 (Additional file 4). Among these pathways, carbohydrate metabolism $(2596,11.80 \%)$, translation $(2325,10.57 \%)$ and folding, sorting and degradation (2036, 9.26\%) were the most extensively over-presented pathways. In addition, the metabolic pathways were related to environmental adaptation, transport and catabolism. Overview, amino acid metabolism, translation and signal transduction were involved in almost every aspect of plant life. Furthermore, it was demonstrated that high-informative and wide coverage were obtained from transcriptome sequencing of leaves in $R$. xanthina f. spontanea, and can be used to analyze the gene products of metabolic pathways and information processing pathways at the molecular level.

\section{DEGs in response to low-temperature stress}

In our studies, the amount of unigenes expression among three libraries under low-temperature $\left(23^{\circ} \mathrm{C}, 4^{\circ} \mathrm{C}\right.$ and $\left.-20^{\circ} \mathrm{C}\right)$ was used to carry out comparative analysis of differences, which was shown significantly difference (Padj $<0.05$, Fig. 5). 358 DEGs (3031 up-regulated and 3891 down-regulated); 42 DEGs (867 up-regulated and 1763 down-regulated); 279 DEGs (2869 up-regulated and 2872 down-regulated) responded to low-temperature stress within the $23^{\circ} \mathrm{C}, 4^{\circ} \mathrm{C}$ and $-20^{\circ} \mathrm{C}$ were detected, respectively. And there were 1536 common genes between T1 vs T2 and T1 vs T3; 3977 common genes appeared in T2 vs $\mathrm{T} 1$ and $\mathrm{T} 2$ vs T3, and 1068 common genes existed in T3 vs T1 and T3 vs T2 (Fig. 5; $23^{\circ} \mathrm{C}, 4^{\circ} \mathrm{Cand}-20^{\circ} \mathrm{C}$ was labeled as T1, T2 and T3, respectively). Otherwise, 468 DEGs had been present in above three groups, which can be associated with cold- 
tolerance in single petal $R$. xanthina f. spontanea. Therefore, it was essential to utilize Go functional enrichment analysis and KEGG pathway enrichment analysis of the DEGs.

\section{GO enrichment analysis of the common DEGs}

In the study, to reveal which biological functions are significantly related to the common DEGs we obtained, a GO functionalenrichment analysis was carried out via the agriGO website with a $p$ score cut-off of 0.05 . The results indicated that the DEGs were involved in biological process, cellular component and molecular function occupied $64.84 \%, 9.38 \%, 25.78 \%$, respectively. Consequently, most DEGs were significant correlated with some biological functions. And we found the DEGs were classified into 83 biological process, mainly focused on transcriptional regulation, DNA-template; transcriptome, response to chitin, response to abscisic acid, response to cold, response to water deprivation, ethylene-activated signaling pathway and response to wounding. As to cellular component, the DEGs were involved in chloroplast, chloroplast thylakoid membrane, integral component of plasma membrane, etc. With respect to molecular function, the DEGs mainly focused on transcription factor activity, sequence-specific DNA binding (Fig. 6 and Additional file 5).

\section{KEGG pathway enrichment analysis of the common DEGs}

In order to more precisely investigate variation of metabolic pathways in leaves during low-temperature stress, statistical pathway enrichment analysis for the DEGs was carried out based on KEGG database and 293 DEGs under low-temperature stress were assigned to 85 different KEGG pathways $(p<0.05)$. It consisted of four significant enriched pathways, which was plant-pathogen interaction, starch and sucrose metabolism, plant circadian rhythm and photosynthesis-antenna proteins, respectively (Table 1 and Additional file 6). When the temperature reached $4^{\circ} \mathrm{C}$ and $-20^{\circ} \mathrm{C}$, most DEGs in plant-pathogen interaction and starch and sucrose metabolism pathways were down-regulated. In addition, the genes in plant circadian rhythm pathway were approximately the same with down-regulated and up-regulated. However, seven DEGs in photosynthesis-antenna proteins pathway were shown up-regulated.

Table 1

Summary of KEGG pathway functional annotations for the common DEGs

\begin{tabular}{|c|c|c|c|c|c|c|}
\hline \multirow[t]{3}{*}{ Pathway } & \multirow[t]{3}{*}{ ID code } & \multirow[t]{3}{*}{$\begin{array}{l}\text { The number of DEGs in metabolic } \\
\text { pathway }\end{array}$} & \multicolumn{4}{|c|}{$\begin{array}{l}\text { Number of the common } \\
\text { DEGs }\end{array}$} \\
\hline & & & \multicolumn{2}{|c|}{$\mathrm{T} 2-\mathrm{T} 1$} & \multicolumn{2}{|c|}{ T3-T1 } \\
\hline & & & Up & down & Up & Down \\
\hline Plant-pathogen interaction & ko04626 & 32 & 6 & 26 & 4 & 28 \\
\hline Starch and sucrose metabolism & ko00500 & 21 & 4 & 17 & 3 & 18 \\
\hline Circadian rhythm - plant & ko04712 & 8 & 3 & 5 & 4 & 4 \\
\hline $\begin{array}{l}\text { Photosynthesis - antenna } \\
\text { proteins }\end{array}$ & ko00196 & 7 & 7 & 0 & 7 & 0 \\
\hline
\end{tabular}

\section{qRT-PCR validation of the DEGs}

Based on the results of transcriptome annotation under low-temperature stress, eight DEGs were screened relating to coldresistant (Fig. 7 and Additional file 7). The expression fold change was analyzed by the use of qRT-PCR before and after low-

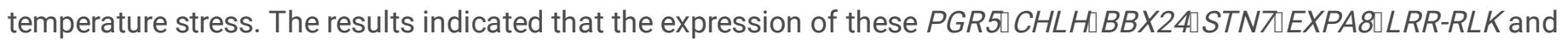
CIPK12 unigenes were up-regulated, one of $b Z I P 60$ was down-regulated, which the same trend was observed in analysis of high-throughput sequencing. Consequently, these genes mentioned above may be a direct correlation with cold-resistant in $R$. xanthina f. spontanea, and the results further confirmed reliability of our transcriptome data. 


\section{Discussion}

In view of the lack of the whole genome-wide data in non-model plants, high- throughput transcriptome sequencing technology can determine each transcriptional fragment sequence and rare transcript, which can also carry out transcriptome analysis for any species, there is no need to understand genetic information of the species. Thus, it is crucial to study non-model plant. In the present study, the transcriptional databases we obtained was compared with known protein databases (Nr, Swiss-Prot, COG, KEGG), according to the principle of "gene structure is similar, function is homologous". As the support of powerful bio-informatics platform, the functional genes were annotated. So far, it has been sequenced successfully from the previous reports in non-model plants [23-27], which provides abundant genetic data sources for plant functional genomics. High-throughout sequencing was performed on the leaves of R.multiflora under low-temperature stress using Illumina Hiseq ${ }^{\text {TM }} 4000$ and a total of 55,084 unigenes were identified. Of these unigenes, 37,303 unigenes (67.7\%) had functional annotation contrasted with $\mathrm{Nr}$ database, and the most frequent plant was Fragaria_vesca, indicated $53.5 \%$. The results consistent with those from the previous studies $[12,28]$, which occupied $32.8 \%$ in R.multiflora and $64.6 \%$ in R.beggeriana Schrenk, respectively.

In the present study, down-regulated genes are more than up-regulated under $4^{\circ} \mathrm{C}$ and $-20^{\circ} \mathrm{C}$ in $R$. xanthina f. spontanea. Previous studies [12] has suggested that up-regulated genes were more than down-regulated with low-temperature stress $\left(4^{\circ} \mathrm{C}\right)$, but the results were different $\left(-20^{\circ} \mathrm{C}\right)$. Therefore, it was essential to confirm the conclusion that high cold-resistance derived from DEGs up-regulated in future. And, our studies indicated that the whole DEGs of up-/down-regulated under $20^{\circ} \mathrm{C}$ stress were less than $4^{\circ} \mathrm{C}$ stress. In contrast to previous studies [12], the results we obtained were not fully concordant maybe the difference of materials, a large number of related genes were expressed by cold signal in the early stage of low temperature in $R$. xanthina f. spontanea.

In the present study, it belonged to biological process (67.8\%) by GO functional enrichment analysis among the assigned DEGs of three common groups when response to low-temperature stress in $R$. xanthina f. spontanea. The results were mainly focused on regulation of transcription, DNA-template, transcription, response to chitin, response to abscisic acid, response to cold, response to water deprivation, ethylene-activated signaling pathway, response to wounding etc., indicating that the DEGs response to low-temperature stress were mainly involved in the biological process of stress and signal transduction.

Based on a KEGG pathway enrichment analysis, all of the common DEGs were annotated in functional categories of plantpathogen interaction, starch and sucrose metabolism, plant circadian rhythm and photosynthesis-antenna proteins. The results consistent with previous reports, all the DEGs were assigned to starch and sucrose metabolism and plant-pathogen interaction under $4^{\circ} \mathrm{C}$ stress in R.beggeriana Schrenk [28]. In the present study, four KEGG metabolic pathways enriched have been proved to be related with cold-resistance in different plants. However, there exist similarities and differences in terms of expression levels for the DEGs contrast with previous studies, and it is essential to further study for its regulatory mechanism.

\section{Conclusions}

Our study first reported response to cold stress at the transcriptome level in Rosa xanthina f. spontanea, which can provides a theoretical basis for further studies on the molecular mechanism of cold-resistance in rose. In the present study, a total of 37,303 unigenes were successfully annotated in $R$. xanthina f. spontanea database, and the results consistent with those of previous studies involved in R.multiflora (32.8\%) and in R.beggeriana Schrenk (64.6\%), respectively. The expression of these PGR5 CHLH BBX24\STNד EXPA8 LRR-RLK and CIPK12 unigenes were up-regulated, one of bZIP60 was down-regulated in Rosa xanthina f. spontanea. The expression trend of eight DEGs selected in this study may be benefit for further studies on cold tolerance mechanisms in rose and other plants.

\section{Methods}




\section{Plant materials and low-temperature treatment}

R. xanthina f. spontanea was collected from Liaoning Research Institute of Cash Crops, Liaoyang, China $\left(41.5^{\circ} \mathrm{N}, 123.8^{\circ} \mathrm{E}\right)$, and its information in details was from Help Me Find (https://www.helpmefind.com/gardening/l.php?l=2.46960.1). Cutting propagation were performed in the greenhouse, annual cutting seedlings with same growth vigor and management conditions, in which were placed variable temperature $\left(23^{\circ} \mathrm{C}\right)$ climate chest one week ago (6h light, $8 \mathrm{~h}$ darkness and $70 \%$ relative humidity), and dropped $2^{\circ} \mathrm{C}$ per hour till to $4^{\circ} \mathrm{C},-20^{\circ} \mathrm{C}$, respectively. Keep this temperature for $12 \mathrm{~h}$, then heat up $2^{\circ} \mathrm{C}$ per hour to $23^{\circ} \mathrm{C}$. The blades were quickly placed in liquid nitrogen, and stored at $-80^{\circ} \mathrm{C}$ refrigerator. All the experiments were repeated three times.

\section{RNA extraction, library construction and sequencing}

Total RNA was extracted using trizol reagent (Invitrogen, CA, USA) following the manufacturer's procedure. The total RNA quantity and purity were analyzedby Bioanalyzer 2100 and RNA 6000 Nano LabChip Kit (Agilent, CA, USA) with RIN number > 7.0. Approximately $10 \mathrm{ug}$ of total RNA representing a specific adipose type was subjected to isolate Poly (A) mRNA purification with poly-T oligo-attached magnetic beads (Invitrogen, CA, USA). Following purification, the poly(A) or poly(A) + RNA fraction was fragmented into small pieces using divalent cations under elevated temperature. Then the cleaved RNA fragments were reverse-transcribed to create the final cDNA library in accordance with the protocol for the mRNA-Seq sample preparation kit (Illumina, San Diego, USA), the average insert size for the paired-end libraries was $300 \mathrm{bp}( \pm 50 \mathrm{bp})$. Then the paired-end sequencing was performed by an Illumina Hiseq 4000 (LC-Bio, China) following the vendor's recommended protocol.

\section{De novo assembly, unigene annotation and differential expression analysis}

Firstly, De novo assembly, functional annotation and classification of the unigenes were performed, cutadapt [16] and inhouse perl scripts were used to remove the reads that contained adaptor contamination, low quality bases and undetermined bases. Then sequence quality was verified using FastQC (http://www.Bioinformatics. babraham.ac.uk/projects/fastqc/), including the Q20, Q30 and GC-content of the clean data. All downstream analyses were based on the clean data with high quality. De novo assembly of the transcriptome was performed with Trinity 2.4 .0 [17]. Trinity groups transcripts into clusters based on shared sequence content.

All assembled unigenes were aligned against NCBI non-redundant protein sequences $(\mathrm{Nr})$ database (http://www.ncbi.nlm.nih.gov/), GO (http://www.geneont ology.org), SwissProt (http://www.expasy.ch/sprot/), KEGG (http://www.genome.jp/ kegg/) and eggnog (http://eggnogdb.embl. de/) databases using DIAMOND [18] with a threshold of E value $<0.00001$.

Differentially expressed unigene analysis Salmon [19] was used to perform expression level for unigenes by calculating transcripts per million (TPM) [20]. The DEGs were selected with log2 (fold change) $>1$ or log2 (fold change) <-1 and with statistical significance $(p<0.05)$ by R package edgeR [21]. Next, GO and KEGG enrichment analyses were again performed based on the DEGs by perl scripts in-house.

\section{qRT-PCR validation}

The leaves of $R$. xanthina f. spontanea were collected and treated at $23^{\circ} \mathrm{C}, 4^{\circ} \mathrm{C}$ and $-20^{\circ} \mathrm{C}, 10 \mathrm{DEG}$ selected at random were used for qRT-PCR validation. Total RNA was extracted by using polysaccharide polyphenol Plant RNA Isolation Kit (N1005, Biobase Technologies Co., Ltd, ChengDu, China), and reverse transcription synthesis cDNA was performed adopting TUREscript 1st Stand cDNA Synthesis Kit (Aidlab Biotechnologies Co., Ltd, Beijing, China). Primers were designed using the Beacon designer 7.9 software for qRT-PCR and listed (Table 2). The qRT-PCR assays were performed on the Analytik JenaqTOWER2.2 (Germany) with the2×SYBR® Green SuperMix (DF, China),and amplified with $1 \mu \mathrm{L}$ of cDNA template, $5 \mu \mathrm{L}$ of 2 $\times$ SYBR Green Super Mix, and $0.5 \mu \mathrm{L}$ of each primer, to a final volume of $10 \mu \mathrm{L}$ by adding water. The amplification program 
consisted of one cycle at $95^{\circ} \mathrm{C}$ for $3 \mathrm{~min}$, followed by 59 cycles of $95^{\circ} \mathrm{C}$ for $30 \mathrm{~s}$ and $60^{\circ} \mathrm{C}$ for $30 \mathrm{~s}$. The relative expression levels were calculated by the $2^{-\triangle \triangle C T}$ method [22].

Table 2

The primers used for qRT-PCR in this study

\begin{tabular}{|lllll|}
\hline No. & Gene ID & Code & Forward primer $(\boldsymbol{F})$ & Reverse primer (R) \\
\hline 1 & TRINITY_DN25157_c0_g1 & PGR5 & AGGGCACAACCCATGATGAA & TTCGGCTCTTAGACAAAGGCAA \\
\hline 2 & TRINITY_DN31568_c1_g5 & CHLH & AGATGAGCCAGTTGAACAGAA & AGTAGGAGCCTGAAGCATTG \\
\hline 3 & TRINITY_DN27059_c1_g3 & BBX24 & CAATAGCCTCTCTGCCAACCA & GGTGGCTCTACGCTACTTGTT \\
\hline 4 & TRINITY_DN30314_C0_g1 & STN7 & CCAGATGGGTTACTCGGCTAA & GACTTCTTCTTAGGCTTCGTTTCC \\
\hline 5 & TRINITY_DN30720_C0_g10 & EXPA8 & GGGTCTTGTTACGAAATGCGATGT & CAGAAGTTGGTGGCGGTGAC \\
\hline 6 & TRINITY_DN32852_c1_g1 & LRR- & GGACCGAGACCTCAATGCTAAGA & TCCTGGTGCTAATGTGAGTGTTCT \\
\hline 7 & TRINITY_DN29285_c0_g3 & CIPK12 & CGAAGAACAACCCGCTCCTCCTC & CGCCCTCGTCGGTCTTGATGT \\
\hline 8 & TRINITY_DN31926_c0_g6 & bZIP60 & TCTTCGTCGTCGTCGTCATC & TTCATCAGCATGTCCTCAACCT \\
\hline 9 & Actin & & CCTCTATGCCAGTGGTCGTACAA & GCCAGGTCAAGTCGCAGAATG \\
\hline 10 & 18s & & CAACCATAAACGATGCCGA & AGCCTTGCGACCATACTCC \\
\hline
\end{tabular}

\section{Abbreviations}

DEGs: Differentially Expressed Genes; KEGG: Kyoto Encyclopedia of Genes and Genomes; qRT-PCR: Quantitative Real-time PCR; GO: Gene Ontology

\section{Declarations}

\section{Ethics approval and consent to participate}

Plant materials (Rosa xanthina f. spontanea.) used in this study were collected from our experimental base (Liaoning Research Institute of Cash Crops, Liaoyang, Liaoning, 110161, China). All plant materials used in this experiment were not endangered materials or species. Study complies with relevant institutional, national, and international guidelines and legislation.

\section{Consent for publication}

Not applicable.

\section{Availability of data and materials}

All data generated or analyzed during this study are included in this published article and its supplementary information files.

\section{Competing interests}

The authors declare that they have no competing interests.

\section{Funding}

This work was financially supported by Doctoral Research Fund Program of Inner Mongolia University for Nationalities (grant no. BS561). 


\section{Authors' contributions}

JJL and XFY conceived and designed research; DFZ conducted the experiments; LX collected and analyzed the data; DFZ wrote the manuscript; $C M$ and ZL provided valuable suggestions on the manuscript; CW and XFY revised the manuscript. All the authors read and approved the manuscript.

\section{Acknowledgements}

Not applicable.

\section{References}

1. Lata C, Prasad M. Role of DREBs in regulation of abiotic stress responses in plants. J Exp Bot. 2011;62: 4731-4748.

2. Theocharis A, Clement C, Barka EA. Physiological and molecular changes in plants grown at low temperatures. Planta. 2012;235: 1091-1105.

3. Wang Z, Gerstein M, Snyder M. RNA-Seq: a revolutionary tool for transcriptomics. Nat Rev Genet. 2009;10: 57-63.

4. Samuel PH, Wu YJ, Joel AK. Gene expression profiling of plant responses to abiotic stress. Functional \& Integrative Genomics. 2003;3(3):105-111.

5. Wang R, Wang X, Liu K, Zhang XJ, Zhang LY, Fan SJ. Comparative transcriptome analysis of halophyte Zoysia macrostachya in response to salinity stress. Plants. 2020; 9: 458-475.

6. Wang XC, Zhao QY, Ma CL, Zhang ZH, Cao HL, Kong YM, Yue C, Hao XY, Chen L, Ma JQ, Jin JQ, Li X, Yang YJ. Global transcriptome profiles of Camellia sinensis during cold acclimation. BMC Genomics. 2013;14: 415-428.

7. Chen JH, Tian QQ, Pang T, Jiang LB, Wu RL, Xia XL, Yin WL.Deep- sequencing transcriptome analysis of low temperature perception in a desert tree, Populus euphratica. BMC Gen. 2014; 15: 326-340.

8. Han Y, Wan HH, Cheng TR, Wang J, Yang WR, Pan HT, Zhang QX. Comparative RNA-seq analysis of transcriptome dynamics during petal development in Rosa chinensis. Scientific Reports. 2017;7: 43382.

9. Huang XL, Yan HQ, Zhai LS, Yang ZT, Yi Y. Characterization of the Rosa roxbunghii tratt transcriptome and analysis of MYB genes. Plos One. 2019; 14: e0203014.

10. Qi WC, Chen X, Fang PH, Shi SC, Li JJ, Liu XT, Cao XQ, Zhao N, Hao HY, Li YJ, Han YJ, Zhang Z. Genomic and transcriptomic sequencing of Rosa hybrida provides microsatellite markers for breeding, flower trait improvement and taxonomy studies. BMC Plant Biology. 2018;18: 1-12.

11. Yan X, Zhang X, Lu M, He Y, An H. De novo sequencing analysis of the Rosa roxburghii fruit transcriptome reveals putative ascorbate biosynthetic genes and EST-SSR markers. Gene. 2015;561: 54-62.

12. Zhang XY, Zhang JZ, Zhang WW, Yang T, Xiong Y, Che DD. Transcriptome sequencing and de novo analysis of Rosa multiflora under cold stress. Acta Physiol. Plant. 2016;38: 164-176.

13. Zhang F, Ji SJ, Wei BD, Cheng SC, Wang YJ, Hao J, Wang SY, Zhou Q. Transcriptome analysis of postharvest blueberries (Vaccinium corymbosum 'Duke') in response to cold stress. BMC Plant Bio. 2020; 20(2):493-501.

14. Michele VR, Laura VR, Ahmed S, Lucimara K, Steven JC, Bernanda C, Patrícia OP, Schuyler SK.Genome-wide expression of low temperature response genes in Rosa hybrida L.. Plant Physiology and Biochemistry. 2020; 146:238-248.

15. Ma Y, Chen JY. A study to evaluate cold hardiness of some parent plants and hybrid seedlings during breeding new cultivars for establishing a rejuvenation rose group. Journal of Beijing Forestry University. 1992;1: 60-65. (In Chinese with English abstract)

16. Martin M. Cutadapt removes adapter sequences from high-throughput sequencing reads. Embnet Journal. 2011; 17: 1012.

17. Grabherr MG, Haas BG, Yassour M, Levinet JZ, Thompson DA. Full-length transcriptome assembly from RNA-seq data without a reference genome. Nature Bio. 2011;29: 644-652. 
18. Buchfink B, Xie C, Huson DH. Fast and sensitive protein alignment using diamond. Nature Methods. 2015;12: 59-60.

19. Patro R, Duggal G, Love MI, Irizarry RA, Kingsford C. Salmon provides fast and bias-aware quantification of transcript expression. Nature Methods. 2017;14: 417-419.

20. Mortazavi A, Williams BA, McCue K, Schaeffer L, Word B. Mapping and quantifying mammalian transcriptomes by RNAseq. Nature Methods. 2008;5: 621-628.

21. Robinson MD, McCarthy DJ, Smyth GK. EdgeR: a bioconductor package for differential expression analysis of digital gene expression data. Bioinformatics. 2010;26: 139-140.

22. Livak KJ, Schmittgen TD. Analysis of relative gene expression data using real-time quantitative PCR and the $2^{-\triangle \triangle C T}$ method. Methods. 2001; 25: 402-408.

23. Arslan M, Devisetty UK, Porsch M, Grosse I, Michalski SG.RNA-Seq analysis of soft rush (Juncus effusus): transcriptome sequencing, de novo assembly, annotation, and polymorphism identification. BMC Gen. 2019; 20: 489-500.

24. Du F, Wu Y, Zhang L, Li X, Zhao X, Wang W, Gao Z, Xia Y. De novo assembled transcriptome analysis and SSR markers development of a mixture of six tissues from Lilium Oriental hybrid 'Sorbonne'. Plant Mol Biol Rep.2015;33: 281-293.

25. Hyun TK, Lee S, Rim Y, Kumar R, Han X, Lee SY, Lee CH, Kim JY. De-novo RNA sequencing and metabolite profiling to identify genes involved in anthocyanin biosynthesis in Korean black raspberry (Rubus coreanus Miquel). Plos One. 2014;9: e88292.

26. Lateef A, Prabhudas SK, Natarajan P. RNA sequencing and de novo assembly of Solanum trilobatum leaf transcriptome to identify putative transcripts for major metabolic pathways. Scientific Reports. 2018; 8: 76-80.

27. Younesi-Melerdi E, Nematzadeh GA, Pakdin-Parizi A, Bakhtiarizadeh MR, Motahari SA. De novo RNA sequencing analysis of Aeluropus littoralis halophyte plant under salinity stress. Scientific Reports. 2020; 10:1-14.

28. Liu Q.Physiological mechanisms and transcriptome analysis on cold resistance of Rosa beggeriana Schrenk. Master's thesis. Chinese Academy of Agricultural Sciences, Beijing. 2017. (In Chinese with English abstract)

\section{Figures}




\section{Length Distribution of Genes}

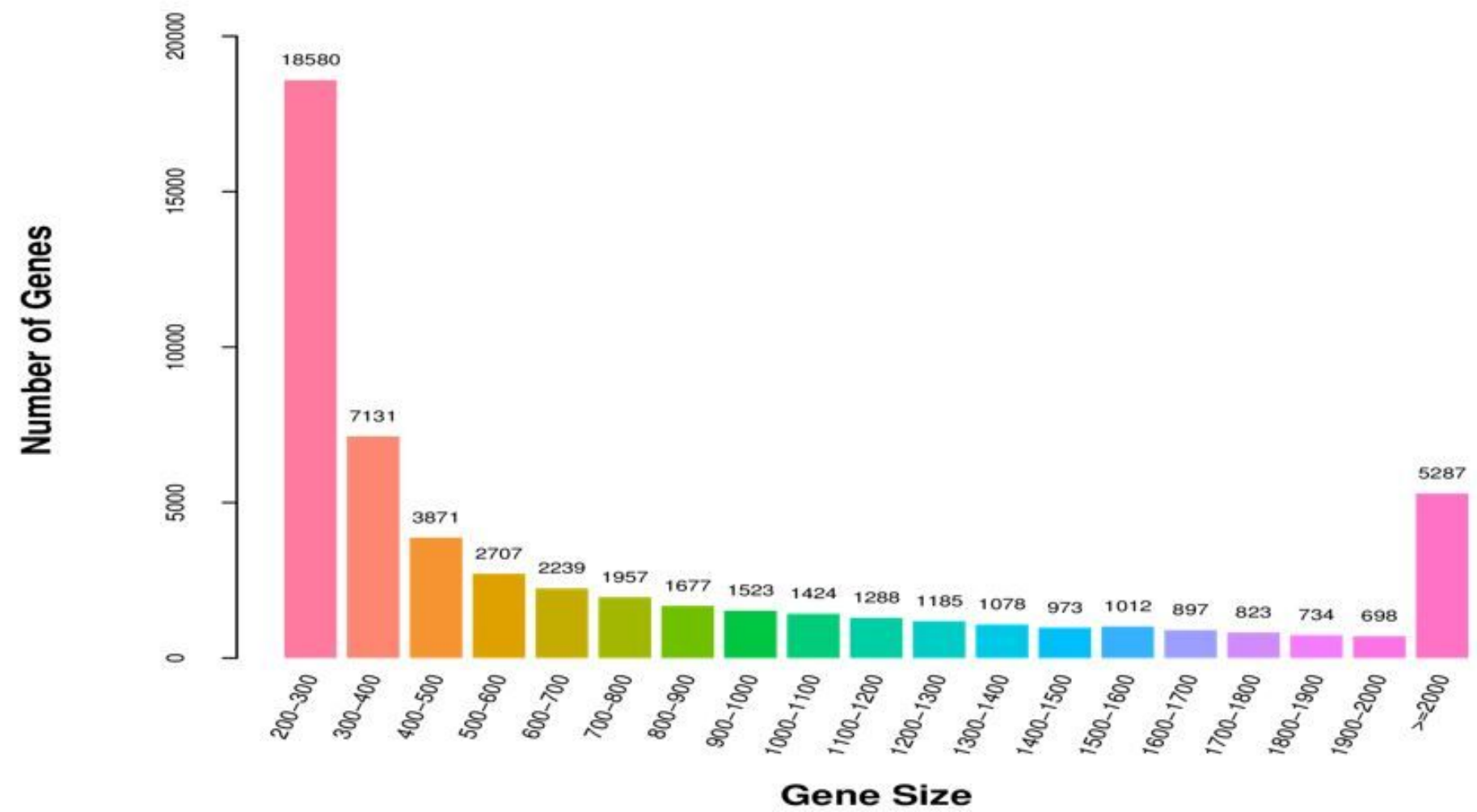

Figure 1

Length distribution of the assembled unigenes in R. xanthina f. spontanea.Non-redundant unigenes were obtained with the length of corresponding gene size ranged from $200 \mathrm{bp}$ to more than $2000 \mathrm{bp}$ 


\section{Species Distribution}

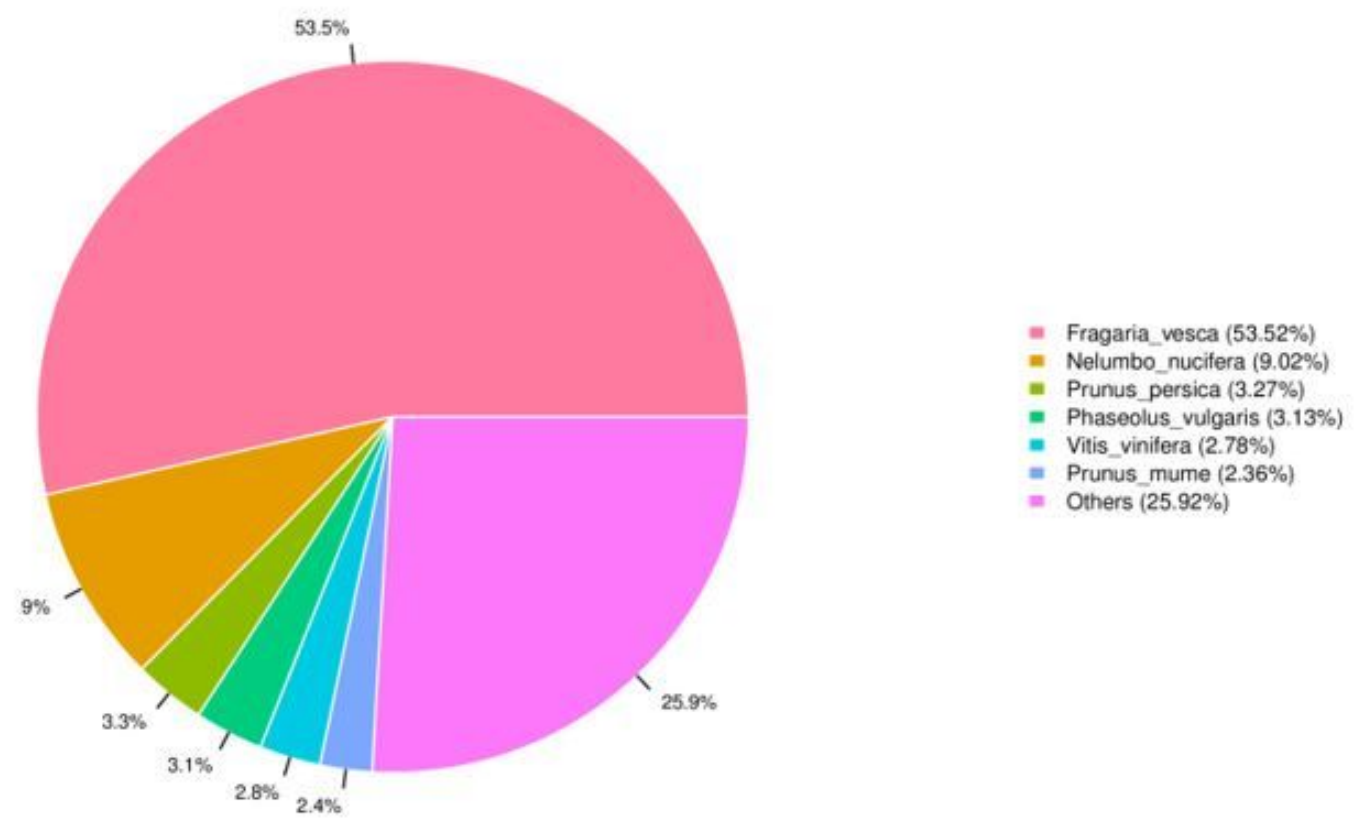

Figure 2

Species distribution of the BLAST hits for each unigenes based on NR database. 


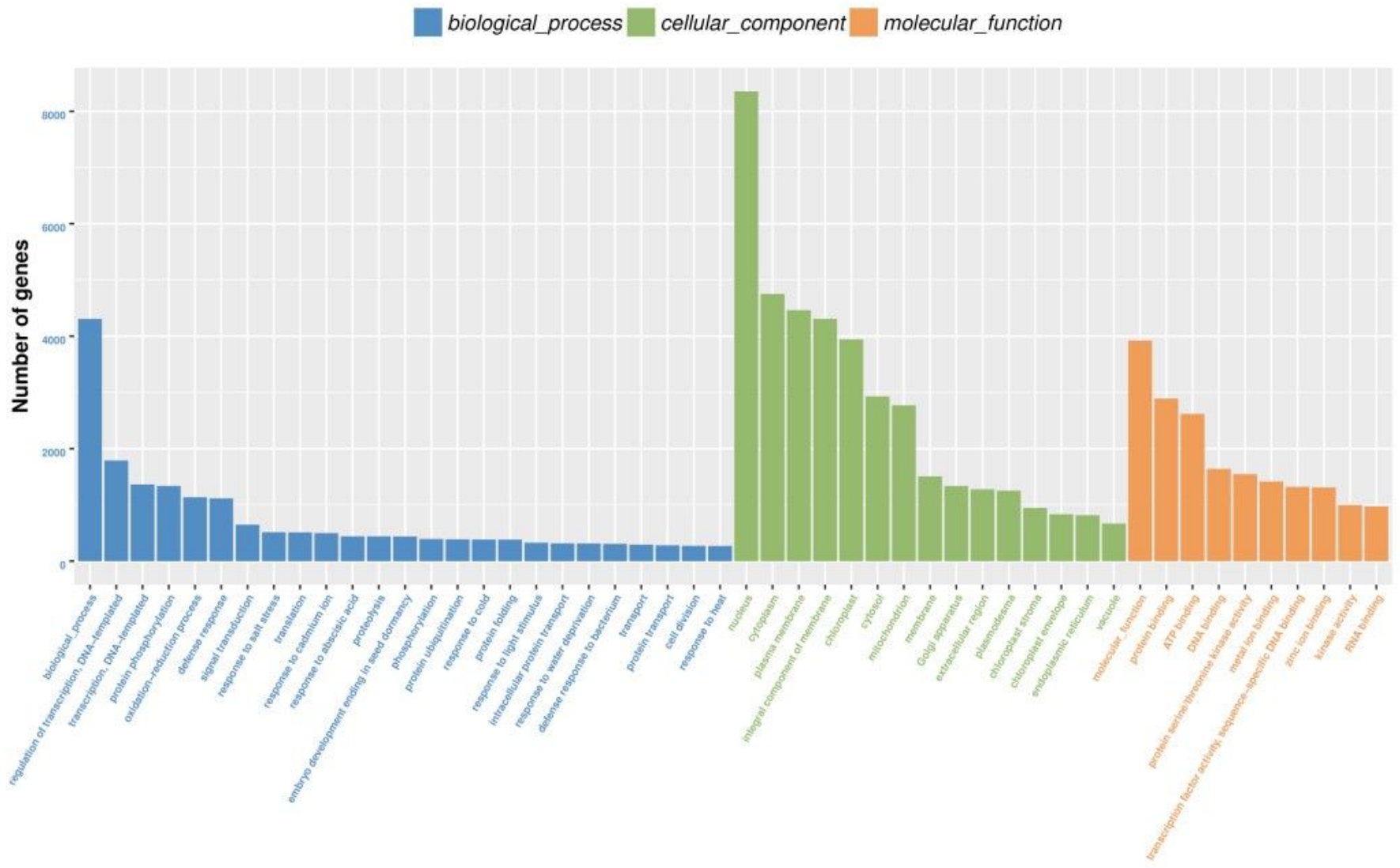

GO terms

\section{Figure 3}

GO enrichment analysis of the assembled unigenes. The unigenes were classified into three main categories: biological process, cellular component and molecular function 


\section{KEGG Pathway Classification}

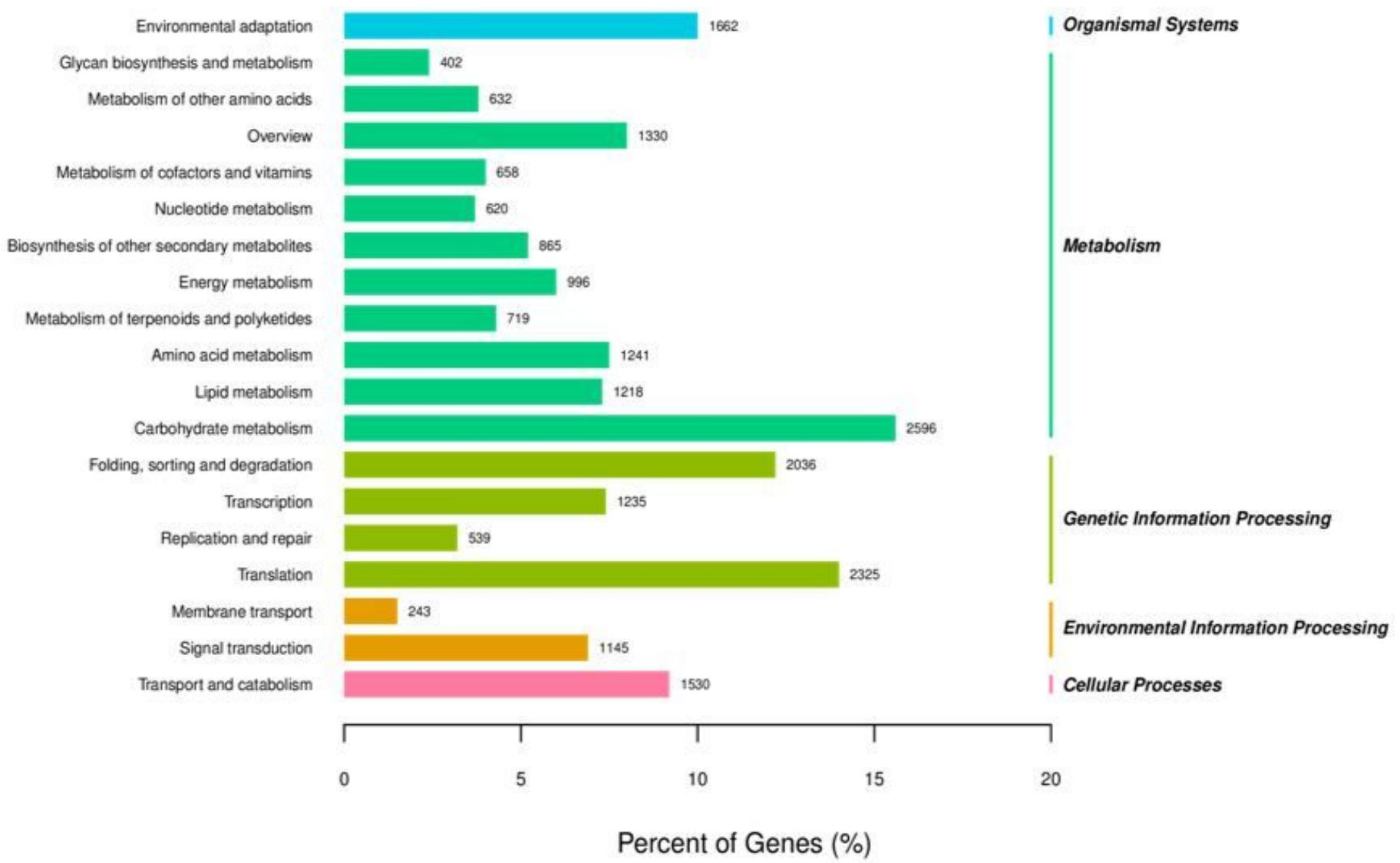

\section{Figure 4}

KEGG pathway functional category of the unigenes assigned in R. xanthina f. spontanea 


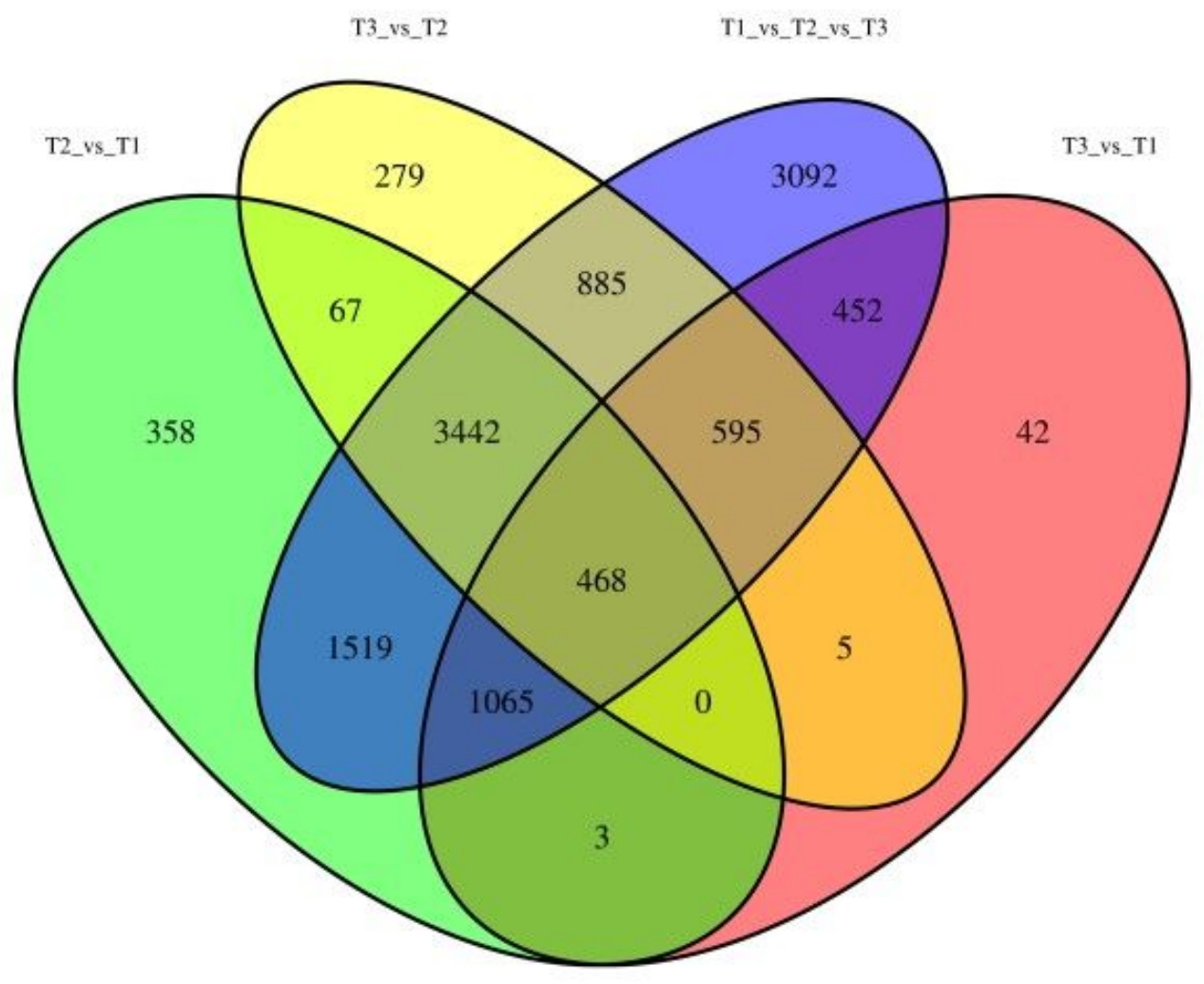

\section{Figure 5}

Venn diagrams of DEGs assembled under low-temperature stress across four sets of comparisons expressed as T3 vs. T1, $\mathrm{T} 2$ vs. T1, T3 vs. T2 and T1 vs. T2 vs.T3, which T1 (control), T2, T3 was noted as $23^{\circ} \mathrm{C}, 4^{\circ} \mathrm{C},-20^{\circ} \mathrm{C}$, respectively (Padj $\left.<0.05\right)$ 


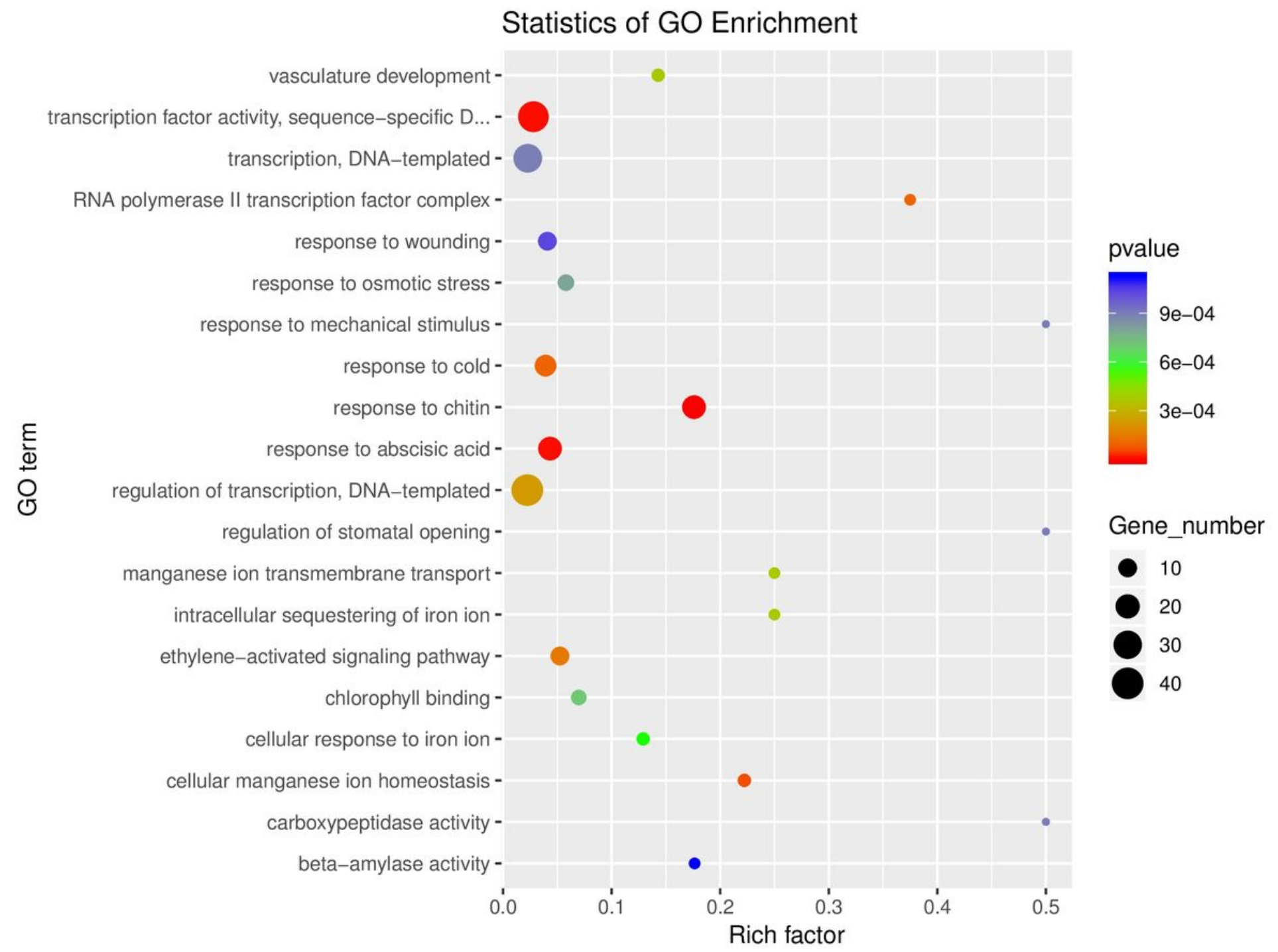

\section{Figure 6}

GO enrichment scatter diagram of the DEGs. The number of genes in each category is equal to the dot size. The dot color represents the corrected $\mathrm{p}$-value 

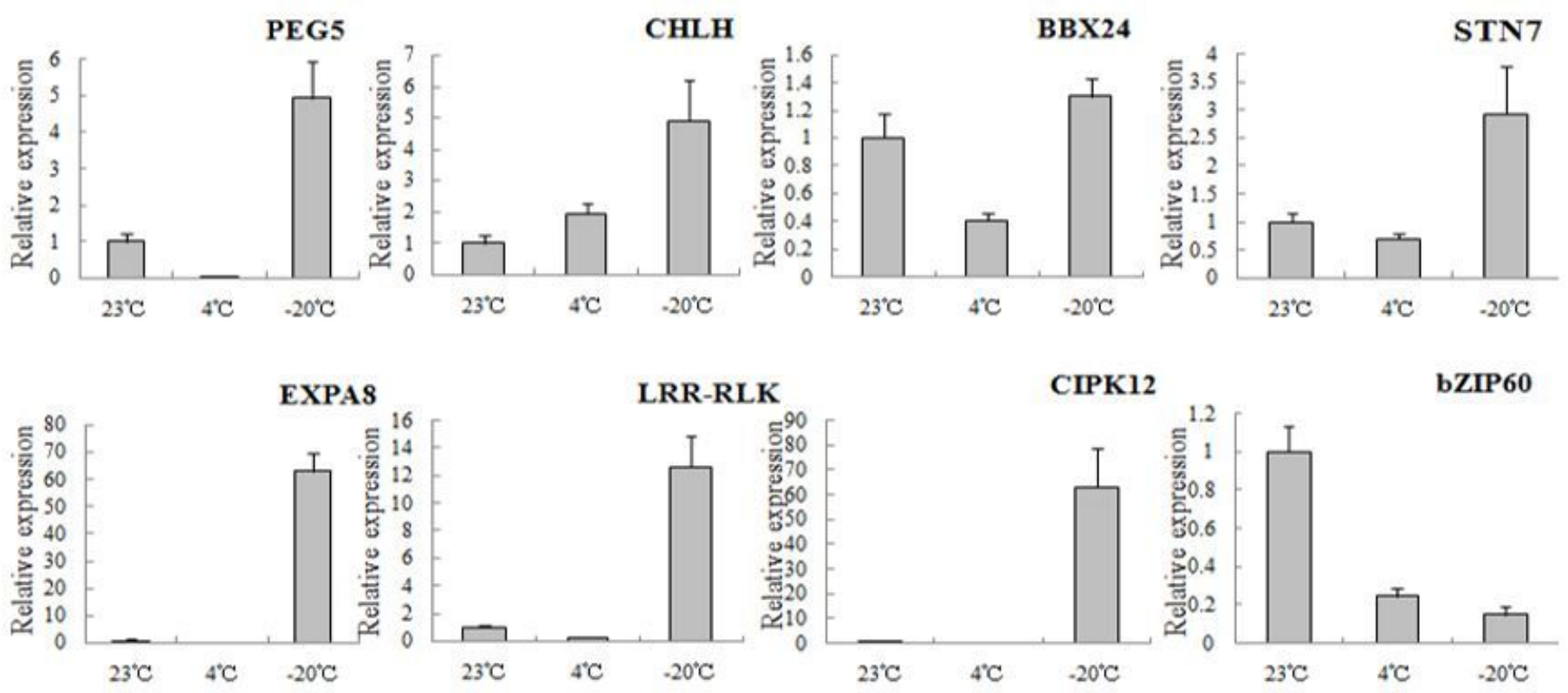

Figure 7

qRT-PCR analysis of expression levels of randomly selected genes in R. xanthina f. spontanea along with corresponding results under $4^{\circ} \mathrm{C},-20^{\circ} \mathrm{C}$ and $23^{\circ} \mathrm{C}$ stress, respectively. Standard errors (SE) bars are shown within each of the columns

\section{Supplementary Files}

This is a list of supplementary files associated with this preprint. Click to download.

- Additionalfile1.xlsx

- Additionalfile2.xlsx

- Additionalfile3.xlsx

- Additionalfile4.xlsx

- Additionalfile5.xIsx

- Additionalfile6.xlsx

- Additionalfile7.xlsx

- Supplementaryinformation.docx 